\title{
28 Research Square \\ Contextual and individual determinants of tooth loss in adults: a multilevel study
}

\section{Luana Leal Roberto ( $\sim$ luleal15@yahoo.com.br)}

Universidade Estadual de Montes Claros https://orcid.org/0000-0003-0940-4733

\section{Marise Fagundes Silveira}

Universidade Estadual de Montes Claros

Alfredo Mauricio Batista De Paula

Universidade Estadual de Montes Claros

\section{Efigênia Ferreira Ferreira}

Universidade Federal de Minas Gerais

Andréa Maria Eleutério De Barros Lima Martins

Universidade Estadual de Montes Claros

Desirée Sant'Ana Haikal

Universidade Estadual de Montes Claros

\section{Research article}

Keywords: Adult. Tooth Loss. Health inequalities. Multilevel Analysis.

Posted Date: February 24th, 2020

DOI: https://doi.org/10.21203/rs.2.16033/v4

License: (c) (i) This work is licensed under a Creative Commons Attribution 4.0 International License. Read Full License

Version of Record: A version of this preprint was published at BMC Oral Health on March 17th, 2020. See the published version at https://doi.org/10.1186/s12903-020-1057-1. 


\section{Abstract}

Background: Tooth loss represents a known marker of health inequality. The association between tooth loss and unfavorable socioeconomic conditions is evident when analyzed at an individual level. However, the effects of contextual characteristics on tooth loss need to be better investigated and understood. The objective of this study was to analyze tooth loss among Brazilian adults (35-44 years of age), in accordance with individual and contextual social characteristics. Methods: This was a multilevel crosssectional study with data from 9,564 adult participants from the Brazilian Oral Health Survey - SBBrasil 2010. The dependent variable was the number of lost teeth and the independent variables were grouped into structural (socioeconomic \& political context) and intermediary (socioeconomic position, behavioral \& biological factors, and health services) determinants. Multilevel Hierarchical Negative Binomial Regression was conducted and the Mean Ratio (MR) was estimated. Results: Brazilian adults lost a mean of 7.57 ( $95 \% \mathrm{Cl} 7.1-8.1)$ teeth. Among the contextual variables, the number of teeth lost was higher among residents of municipalities with high and medium/low Municipal Human Development Index (MHDI) and in municipalities that did not have public water fluoridation. Among the individual variables, dental loss was higher among those who declared themselves yellow/black/brown/indigenous, were older, who had lower income, who had never visited a dentist, who had used dental services for more than a year and those whose most recent visit to the dentist was due to oral health problems. On the other hand, dental loss was lower among adults with higher education levels and males. Conclusions: The number of missing teeth was associated with unfavorable contextual and individual conditions, which reinforces the need to reduce social inequality and guarantee regular, lifetime access to dental services.

\section{Background}

Tooth loss is considered as a major oral health issue [1,2] and an important public health problem [3]. Besides reflecting the accumulation of oral disease throughout life [4], tooth loss can also be influenced by social, behavioral and cultural factors $[3,5]$. Thus, tooth loss is the result of a complex interaction between biological and social factors [6].

Tooth loss is a marker of health disparity in the population [7], based on the fact that socially disadvantaged groups show lower number of the teeth [8]. This association is evident at the individual level $[2,7,9-11]$. However, the effects of the place where people live (contextual effects) on tooth loss needs further investigation to be understood.

The need to recognize and distinguish contextual influences on oral health has been identified in the scientific literature. A systematic review conducted to identify the contextual socioeconomic factors associated with dental loss found that there are very few studies evaluating tooth loss in the contextual perspective [12]. Some studies have reported the contextual characteristics associated with tooth loss, such as Gini coefficient [7, 13], municipal human development index (MHDI) [14], fluoridated water supply [14-16], and place of residence (urban versus rural) [15]. Such studies are essential, since not all determinants of the health-disease process can be captured at the individual level [17]. 
This study aimed to analyze tooth loss among Brazilian adults, and asses its association with both contextual social inequalities and individual characteristics using a multilevel approach. We hypothesized that the total number of missing teeth in adults is affected by contextual social inequalities even after controlling for individual variables related to tooth loss.

\section{Methods}

The study was designed as a cross-sectional multilevel study. In addition to individual variables, contextual variables were taken into account to explain an outcome assessed at the individual level. The individual variables were obtained from the Brazilian Oral Health Survey - SBBrasil 2010 [18], and the contextual variables were collected at the municipal level from official public databases.

SBBrasil 2010 was a national epidemiological survey on oral health funded by the Ministry of Health. For representation of the complete Brazilian population, individuals aged 5 and 12 years and those in age groups 15-19, 35-44, and 65-74 years from 177 Brazilian municipalities were evaluated. Sampling was carried out at different domains of the state capitals, federal district, and municipalities within defined geopolitical macro-regions (North, Northeast, Central West, Southeast, and South), using probabilistic sampling in multiple stages with a design effect (DEFF) of 2. The primary sampling units were: (a) municipality, for the interior of the regions, and (b) census tract for the state capitals. Interviews and oral examinations were conducted in the subjects' homes. Oral examinations were performed under natural light, by trained and calibrated examiners (Kappa $\geq 0.65$ ), using a handheld computer to record the data.

The diagnostic criteria of Oral Health Surveys: Basic methods ( ${ }^{\text {th }}$ edition) from World Health Organization (WHO) were used [19]. In addition to assessment of the individual's oral condition, interview was conducted with each household and comprised questions related to the socioeconomic profile of the family, use of dental services, self-reported oral morbidity, and self-perception of oral health. Details of the methodology used in SBBrasil 2010 have been described in a previous study [20]. In the present study, data of 9,779 individuals in SBBrasil 2010 between the ages of 35 and 44 years were used, which is the standard age group for evaluation of oral health conditions in adults [19].

Contextual variables were collected from official public databases for each of 177 participating municipalities of SBBrasil 2010: Demographic census of 2010 by the Brazilian Institute of Geography and Statistics (IBGE) [21]; Atlas Brazil of the United Nations Development Program (UNDP) [22]; National Survey of Basic Sanitation of IBGE [23]; and the Department of Informatics of the Unified Health System (DATASUS) [24]. In the databases of Atlas Brazil [22] and National Survey of Basic Sanitation [23], data of IBGE 2010 demographic census were acquired between August 1, 2010 and October 30, 2010 from 316,574 census tracts with predefined territorial boundaries [21].

In this study, the dependent variable was total number of missing teeth defined as any natural tooth missing due to extraction, for any reason corresponding to codes 4 and 5 of the DMFT index (decayed, missing and filled teeth) [19]. This was assessed according to its discrete numerical nature whose values are whole numbers (counts). 
The conceptual model for inequities in oral health of Watt \& Sheiham (2012) [25] was building based on Conceptual Framework for Action on the Social Determinants of Health (CSDH) [26]. In our study, that model was used for the grouping of contextual and individual independent variables. This theoretical model takes into account the social determinants of inequalities in oral health, in contrast to preventive approaches, that focus on the behavioral changes of the individual. According to this conceptual model, the variables that influence the oral health can be grouped into structural determinants (socioeconomic \& political context) and intermediary determinants (socioeconomic position, behavioral \& biological factors, and health services) (Figure 1).

In the socioeconomic \& political context, all contextual variables were included: geographical location of the municipality (capital; interior) [18], Municipal Human Development Index (MHDI) (very high; high; medium/low) [22], Gini coefficient [22], percentage of the population with access to garbage collection [21], percentage of the population with access to a bathroom and piped water [21], coverage of oral health teams [24], number of dentists per 1000 inhabitants [24], and public water fluoridation (yes; no) [23]. MHDI reflects composite information on income, education level, and longevity in each municipality, and the scores are on a scale from 0 to 1 , where higher values reflect a higher level of human development. Gini coefficient measures inequality in income distribution on a scale from 0 (absolute equality) to 1 (absolute inequality) [22]. The percentage of the population with access to garbage collection refers to the proportion of the population of each municipality with access to public garbage collection services [21]. The percentage of the population with access to a bathroom and piped water refers to the proportion of households in the municipality with simultaneous access to water supply (running water) by the distribution network, and bathroom or toilet facilities exclusively for residents [21]. The coverage of oral health teams refers to the proportion of the population in the municipalities that receive primary care of oral health teams [24]. The public water fluoridation classification used in this study was performed according to National Survey of Basic Sanitation from IBGE [23], which is exclusively based on information provided by sanitation companies. All contextual variables were analyzed as quantitative data expressed as numbers, except variables of the geographic location of the municipality, MHDI, and public water fluoridation.

In the socioeconomic position, individual variables were included as follows: declared skin color (white; yellow/black/brown/indigenous), education level (years of study), and family income in USD $(>2,557$; $853-2,556 ; 285-852 ; \leq 284)$; and the minimum wage at the time of data collection was USD 290.0 .

In relation to behavioral \& biological factors, individual variables were included as follows: sex (female; male), age (years), self-perception of the need for treatment (yes; no), and pain in the teeth and gums in the last 6 months (no; yes). Also at this level, considering health services, the following individual variables were included: previous use of dental service (yes; no), time since last consultation ( $\leq 1$ year; > 1 year; no previous use of dental service), reason for consultation (review/prevention; oral health problems; no previous use of dental service), type of dental service (public; not public; no previous use of dental service), and satisfaction with dental services (satisfied; dissatisfied; no previous use of dental service). 


\section{Analyses}

To explore the dependent variable, a map was drawn with the average number of lost teeth for each one of the five Brazilian geopolitical macroregions, state capitals, and federal district. For each Brazilian macroregion, besides mean teeth lost, a confidence interval of $95 \%(95 \% \mathrm{Cl})$ was estimated corrected by Deff. Geographic information system (GIS)-based Quantum GIS (QGIS) software (General Public License; $\mathrm{GNU}$ ), which is freely available online, was used for manipulation of spatial data and construction of a map.

The data relating the individual and contextual variables was initially organized in the statistical software Predictive Analytics Software (SPSS/PASW ${ }^{\circledR}$ ) version 18.0 for Windows ${ }^{\circledR}$. The descriptive analyses of the contextual variables used only the municipal data. The descriptive analysis of the individual variables was conducted according to the need of correction for the effect of sample design, because they are from samples by conglomerates. For such, the Complex Samples module was used, considering the weights resulting from the sampling process adopted. Measures of central tendency and variability were estimated for the numerical independent variables and simple (n) and relative (\%) frequencies for categorical independent variables. The association between the total number of lost teeth and the individual characteristics was verified by the non-parametric tests: Spearman correlation ( $\mathbb{\nabla})$ for numerical independent variables; Mann-Whitney test for dichotomous independent variables; and Kruskall-Wallis test for the polytomous independent variables.

The data was exported to the STATA ${ }^{\circledR}$ software, version 14.0, and the Multilevel Hierarchical Negative Binomial Regression (stepwise backward method) model was used with use of contextual and individual data. The Negative Binomial Regression model is appropriate when the dependent variable is quantitative and with non-negative, integer values (counting data) and when there is overdispersion in the data (the variance of the dependent variable is greater than the mean) [27]. Before starting the modeling, the adequacy of the dependent variable for this regression modality was verified and confirmed. For estimation of adjustment between outcome (total number of teeth lost) and the independent variables from first (contextual) and second (individual) levels of analysis, the fixed effect model was used [28]. Initially, an empty model was used with only a random intercept and the dependent variable, without the others variables. Subsequently, a hierarchical block design was used as proposed by the adopted theoretical model [25] (Figure 1). Model 1 included only the contextual variables (socioeconomic \& political context). All eight contextual variables adopted in our study were included in this first model. Adjustment was made in Model 1 and only the contextual variables that were significantly associated with the outcome $(p \leq 0.05)$ were maintained. From the second model, the individual variables were taken into account. Model 2 kept the contextual variables adjusted in model 1, and added the socioeconomic position. This model was also adjusted ( $p \leq 0.05$ ). Model 3 comprised the variables adjusted in models 1 and 2 and added behavioral \& biological factors and health services. This final model was adjusted again ( $\mathrm{p} \leq 0.05)$. The menbreg, irrfunction was used to obtain the Mean Ratio (MR) and its $95 \% \mathrm{Cl}$. Moreover, a sensitivity analysis was performed using a multilevel logistic regression model. In order to accomplish this, a dependent variable number of missing teeth was dichotomized by 
its median (under and above median points). A supplementary file exhibits findings from this analysis (Table S1).

The analysis of the fit of the models was performed through Deviance, obtained through the Log Likelihood multiplied by (-2), where it is expected that there will be significant differences between the models (difference greater than 3.84) [29]. In addition, multicollinearity was tested by verifying the correlations of independent variables, with no values above 0.7 being identified. We also conducted a comparison between both Brazilian adults included and excluded of the final analysis due to losses of the independent variables. A supplementary file shows findings of these analyses (Table S2).

SBBrasil 2010 was conducted according to the ethical principles of the Resolution of the National Health Council (CNS; number 196/96), related to research on human beings; it was approved by the Research Ethics Committee of the Ministry of Health and registered at the National Research Ethics Committee of Brazil (CONEP) (CNS approval number: 15.498/2009). In all participants, information on the research was provided, and signed informed consent forms were obtained [16].

\section{Results}

Among the 9,779 adult individuals of SBBrasil 2010, 215 (2.2\%) were excluded due to no information of the dependent variable. Finally, a total of 9,564 individuals were included in the study. The average number of adults evaluated in each Brazilian municipality was 54.03 ( \pm 97.92$)$, ranging from 3 to 488 individuals. The median tooth loss was 6.0, while the mean tooth loss was 7.57 (95\% Cl: 7.1-8.1) teeth, with higher values attained in the North (10.95) and Northeast (8.77) regions. Among the state capitals, tooth loss was lower in Vitória, Espírito Santo (4.23), followed in increasing order by Porto Alegre, Rio Grande do Sul (4.29), Belo Horizonte, Minas Gerais (5.03), and Florianópolis, Santa Catarina (5.13) (Figure 2).

In what it refers to contextual variables, among the 177 municipalities analyzed, $85 \%$ were municipalities located in interior regions of the state, one third had no fluoridated water, and the average Gini coefficient was 0.62 ( \pm 0.12 ). Mean tooth loss in adults from interior municipalities was 7.75 , while adult city dwellers exhibited mean tooth loss of 6.45 . In municipalities without fluoridated water, the mean number of missing teeth in adult population was 10.53 , while for municipalities with fluoridated water, this mean was 7.53. The mean number of missing teeth was $5.62,8.61$, and 10.17 in adults from municipalities with very high, high, and medium/low MHDI, respectively.

In the descriptive analysis of the individual variables, there was a predominance of females and those who self-declared themselves as yellow/black/brown/indigenous. The mean age of adult individuals was 39.39 years $( \pm 3.08)$, and the average years of education was $8.64( \pm 3.93)$. Most of the adults utilized public dental services and self-perceived the need for dental treatment (Table 1). The bivariate analysis can also be observed in Table 1. 
The results of multilevel hierarchical negative binomial regression analysis are shown in Table 2. With regard to contextual variables, tooth loss was higher in the municipalities with high or medium/low MHDI score ( $M R=1.34$ and 1.46 respectively). The average dental loss was increased $27 \%$ in the municipalities without fluoridated water, as compared to that in the municipalities with public water fluoridation (MR = 1.27). With regard to individual variables, the average number of tooth loss was higher in declared to be yellow / black / brown / indigenous ( $M R=1.06)$, and lower among those with higher education levels $(M R=0.93)$. The average dental loss was higher in the individuals with family income of $\leq$ USD 2556.00 than in those with family income of $\geq$ USD 2557.00 . In addition, it was lower in the male individuals than in the female individuals, which represents protection against tooth loss in male adults $(M R=0.87)$. The total number of missing teeth was higher among the older individuals ( $M R=1.09)$ who never visited the dentist $(M R=1.42)$, those who received dental service more than 1 year ago $(M R=1.05)$, and those with last visit to the dentist due to oral health problems $(M R=1.42)$. Deviance was significantly reduced among the different models after adding each new block of variables.

\section{Discussion}

A higher tooth loss was observed among the residents of municipalities with high or medium/low MHDI and who did not have public water fluoridation, even after adjustment for individual variables. Among the individual variables, the number of lost teeth was influenced by declared skin color, education levels, income, sex, age, previous use of dental service, time elapsed since the last dental consultation and the reason for this consultation. In general, the highest number of missing teeth was related to unfavorable individual and contextual conditions.

The mean number of tooth loss among Brazilian adults was 7.57. Historically, a reduction in the number of lost teeth among Brazilian adults has been observed [18,30,31]. However, the number of lost teeth remains higher in less developed regions of the country (North and Northeast - $10.95-8.77$ ). It should be emphasized that this pattern of regional differences has remained over time, in all age ranges $[18,30,31]$. In addition, levels of tooth loss among adults in Brazil are still higher than those observed in developed countries such as Canada (6.7) [32], Bulgaria (6.7) [33] and Ireland (5.7) [34]. This variation in the number of lost teeth for the different localities reinforces the idea of contextual influences on tooth loss and validates the findings of our study that unfavorable contexts increase the occurrence of tooth loss.

The contextual variables MHDI and public water fluoridation remained associated with the number of lost teeth, even after adjustment for important individual variables known to be associated to tooth loss. The association between contextual variables and the presence of functional dentition [14,15], number of selfreported teeth $[7,16,32,35]$ and edentulism $[13]$ has already been reported among adults. However, no previous studies have evaluated this relationship considering the number of lost teeth as numerical variable. This option offers the advantage of observing the magnitude of the impact per number of lost teeth, without the need for categorization of the variable, this allows the analysis to be more sensitive. 
Adults living in municipalities with high or medium/low MHDI had a higher number of lost teeth compared to adults living in municipalities that had very high MHDI. Previous studies had already verified the effect of MHDI on the higher prevalence of functional dentition [14] and lower need for dental treatment among adults [36]. Municipalities that have greater MHDI possibly offer better opportunities for the maintenance of oral health, especially through increased access to dental services [35]. Furthermore, it is known that other important aspects for the maintenance of dentition, such as higher education levels, better eating habits, greater access to information and provision of health services, are commonly more available in developed areas [35,37].

The public water fluoridation was associated with a lower number of lost teeth, which corroborates previous studies [14-16]. The effect of water fluoridation in reducing the prevalence and incidence of dental caries [38], the main cause of tooth loss [2], is widely recognized. Although we did not determine the time of availability of fluoride in the water supply to the municipalities, we believe individuals may benefit from access to fluoridated water throughout their life, rather than at a specific time point. The results emphasize the importance of water fluoridation as a public health measure. It was found that the impact of the addition of fluoride in the public water supply is higher for individuals of lower socioeconomic level [39], which reinforces the importance of this measure as a way to compensate for inequalities in oral health [38].

In relation to individual variables, adults with higher education levels and with higher income had lower tooth loss. This association is consistent in the national $[2,3,11,14-16]$ and international literature $[5,7,32,33]$. Adults who declare being yellow, black, brown or indigenous displayed a higher tooth loss compared to whites. A study conducted among Brazilian adults also found racial inequity associated with tooth loss, with greater vulnerability of blacks and browns compared to whites [40]. The variable declared skin color was allocated in the block of socioeconomic position, since genetic studies have identified that there may not be a significant association between skin color and genomic ancestry $[41,42]$. Thus, in countries where there is a large miscegenation, as is the case for Brazil, the variable of declared skin color seems to reflect more a socioeconomic condition than a genetic aspect.

A lower number of tooth loss among men $[2,3,10,11,14-16,33]$ and higher among older individuals $[3,5,11,14-16,33]$ had already been observed in previous studies. Moreover, the number of missing teeth was higher among adults who never used dental services, who used dental services more than a year ago and whose reason for consultation was for oral health problems, to the detriment of preventive use. Previous studies have also reported an association between the use of dental services in the last year and lower tooth loss $[5,14,15]$. The adult population, especially workers, may have difficulties in accessing oral health services during the normal business hours [43]. This possibly leads these individuals to use dental services sporadically, seeking care for urgent dental conditions, in which restorative treatments may not be an option, increasing the likelihood of tooth loss. In addition, the preventive use (review/followup/routine/check-up) of dental services is considered an indicator of oral health, and the most conservative dental treatments were performed in patients who used them regularly [44]. A prospective cohort study carried out in New Zealand found that adults who routinely used dental services had higher 
levels of oral health, with less decayed and lost teeth [45]. Based on these facts, access to dental services on a regular basis should be encouraged in order to reduce the number of tooth loss among adults.

Our study has some limitations that should be highlighted. First, tooth loss was measured crosswise but it reflects the disease accumulation throughout life. In this way, we cannot establish a temporal relationship between tooth loss outcome and the others independent variables investigated in this study. Second, secondary data were analyzed and, consequently, important tooth loss risk factors, such as occurrence of systemic chronic conditions and lifestyle factors were not assessed once SBBrasil (2010) did not measure those characteristics. Longitudinal studies are needed to better clarify this issue. In the other hand, the present study included a representative sample of Brazilian adult population. Moreover, the multilevel approach of the variables allowed identifies the contribution of each block of variables to tooth loss. With this done, it was realized the influence of both individual and contextual factors on tooth loss outcome. Such effects were confirmed by using sensitivity analysis, which even changing the classification of the dependent variable and the type of analysis, it was still found the same contextual variables associated with tooth loss parameter. The random effect to contextual variables and for the individual variable sex was tested. However, there was no note any significant improvement in model adjustment.

\section{Conclusions}

The contextual variables MHDI and public water fluoridation remained associated with the number of lost teeth among adults, even after controlling for important individual variables known to be associated with tooth loss. Generally, a higher number of missing teeth was associated with unfavorable contextual and individual factors: lower MHDI, absence of the public water fluoridation, less education levels, low family income, no previous use of dental service and greater time elapsed since the last dental consultation. Thus, the findings of this study reinforce the impact of social inequality in tooth loss and require a reflection on the need for expansion and better organization of dental services in order to promote equity between individuals.

\section{Declarations}

\section{Ethics approval and consent to participate}

National Research Ethics Committee of Brazil (CONEP) - 15.498/2009

\section{Consent for publication}

Not applicable.

\section{Availability of data and materials}


The datasets used and/or analyzed during the current study are available from the corresponding author on reasonable request.

\section{Competing interests}

The authors declare that they have no competing interests.

\section{Funding}

Not applicable.

\section{Authors' contributions}

LL Roberto carried out the analyses, data interpretation and writing of the paper. MF Silveira contributed in the data analysis. AMB de-Paula and EF Ferreira worked in critical review of the paper. AMEBL Martins worked on the data interpretation and critical review of the paper. DS Haikal acted in the design of the study, contributed in the analysis, data interpretation and critical review of the paper.

\section{Acknowledgements}

LL Roberto has a doctoral scholarship by the Coordination for the Improvement of Higher Education Personnel (CAPES). AMB de Paula, EF Ferreira, and AMEBL Martins are holders of the productivity researcher scholarships form the National Council for Scientific and Technological Development (CNPq).

\section{References}

1. Sanders AE, Slade GD, Turrell G, Spencer AJ, Marcenes W. Does psychological stress mediate social deprivation in tooth loss? J Dent Res. 2007; 86(12): 1166-70.

2. Peres MA, Barbato PR, Reis SCGB, Freitas CHSM, Antunes JLF. Tooth loss in Brazil: analysis of the 2010 Brazilian Oral Health Survey. Rev Saude Publica. 2013; 47(Supl 3): 1-11.

3. Barbato PR, Nagano HCM, Zanchet FN, Boing AF, Peres MA. Perdas dentárias e fatores sociais, demográficos e de serviços associados em adultos brasileiros: uma análise dos dados do Estudo Epidemiológico Nacional (Projeto SB Brasil 2002-2003). Cad Saude Publica. 2007; 23(8): 1803-14.

4. Thomson WM. Social inequality in oral health. Community Dent Oral Epidemiol. 2012; 40(Supl 2): 2832.

5. Hamasha AA, Sasa I, Al-Qudah M. Risk indicators associated with tooth loss in Jordanian adults. Community Dent Oral Epidemiol. 2000; 28(1): 67-72.

6. Tu YK, Gilthorpe MS. Commentary: is tooth loss good or bad for general health? Int J Epidemiol. 2005; 34(2): 475-6. 
7. Bernabé E, Marcenes W. Income inequality and tooth loss in the United States. J Dent Res. 2011; 90(6): 724-9.

8. Chestnutt IG. Adressing oral health inequalities in the United Kingdom: the impact of devolution on population-based fluoride policy. Br Dent J. 2013; 215(1):11-12.

9. Gilbert GH, Duncan RP, Shelton BJ. Social determinants of tooth loss. Health Serv Res. 2003; 38(6 Pt 2): $1843-62$.

10. Susin C, Oppermann RV, Haugejorden O, Albandar JM. Tooth loss and associated risk indicators in an adult urban population from south Brazil. Acta Odontol Scand. 2005; 63(2): 85-93.

11. Haikal DS, Martins AMEBL, Aguiar PHS, Silveira MF, Paula AMB, Ferreira EF. 0 acesso à informação sobre higiene bucal e as perdas dentárias por cárie entre adultos. Cien Saude Colet. 2014; 19(1): 287300 .

12. Barbato PR, Peres KG. Determinantes socioeconômicos contextuais das perdas dentárias em adultos e idosos: uma revisão sistemática da literatura. Rev Bras Epidemiol. 2015; 18(2): 357-71.

13. Celeste RK, Nadanovsky P, Ponce de Leon A, Fritzell J. The individual and contextual pathways between oral health and income inequality in Brazilian adolescents and adults. Soc Sci Med. 2009; 69(10): 1468-75.

14. Chalub LLFH, Martins CC, Ferreira RC, Vargas AMD. Functional Dentition in Brazilian Adults: An Investigation of Social Determinants of Health (SDH) Using a Multilevel Approach. PLoS One. 2016; 11(2): e0148859.

15. Koltermann AP, Giordani MA, Pattussi MA. The association between individual and contextual factors and functional dentition status among adults in Rio Grande do Sul State, Brazil: a multilevel study. Cad Saude Publica. 2011; 27(1): 173-82.

16. Barbato PR, Peres MA, Höfelmann DA, Peres KG. Indicadores contextuais e individuais associados à presença de dentes em adultos. Rev Saude Publica. 2015; 49:27.

17. Diez-roux AV. The study of group-level factors in epidemiology: rethinking variables, study design, and analytical approaches. Epidemiol Rev. 2004; 26: 104-11.

18. Ministério da Saúde. Departamento de Atenção Básica. SBBrasil 2010. Pesquisa nacional de saúde bucal: Resultados principais. Brasília: Ministério da Saúde, 2012.

19. World Health Organization (WHO). Oral health surveys: basic methods. 4th Ed. Geneva: World Health Organization; 1997. 
20. Roncalli AG, Silva NND, Nascimento AC, Freitas CHSDM, Casotti E, Peres KG, et al. Aspectos metodológicos do Projeto SBBrasil 2010 de interesse para inquéritos nacionais de saúde. Cad Saúde Pública. 2012; 28(Suppl): 40-57.

21. Instituto Brasileiro de Geografia e Estatística. Síntese de indicadores sociais: uma análise das condições de vida da população brasileira. Estudos e Pesquisas. 2011; 27: 1-4.

22. Atlas do Desenvolvimento Humano no Brasil. 2010. http://www.atlasbrasil.org.br/2013/pt/consulta. Accessed 06 jan 2019.

23. Instituto Brasileiro de Geografia e Estatística. Pesquisa Nacional de Saneamento Básico. http://www.sidra.ibge.gov.br/bda/pesquisas/pnsb/. Accessed 06 jan 2019.

24. Ministério da Saúde. Informações de saúde. Informação de saúde: cadernos de informação de saúde. 2010. http://tabnet.datasus.gov.br/tabdata/cadernos/cadernosmap.htm. Accessed 15 fev 2019.

25. Watt RG, Sheiham A. Integrating the common risk factor approach into a social determinants framework. Community Dent Oral Epidemiol. 2012; 40(4): 289-96.

26. World Health Organization (WHO). A Conceptual Framework for Action on the Social Determinants of Health. Geneva: World Health Organization; 2010.

27. Fávero LP. Análise de Dados - Modelos de Regressão Com Excel ${ }^{\circledR}$, Stata ${ }^{\circledR}$ e Spss ${ }^{\circledR}$. Rio de Janeiro: Editora Elsevier; 2015.

28. Snijders TAB. Multilevel Analysis International Encyclopedia of Statistical Science. Berlin, Heidelberg: Springer Berlin Heidelberg; 2011. p. 879-82.

29. Carle AC. Fitting multilevel models in complex survey data with design weights: Recommendations. BMC Medical Research Methodology. 2009; 9:49.

30. BRASIL - Ministério da Saúde (BR). Secretaria Nacional de Programas Especiais de Saúde. Fundação de Serviços de Saúde Pública. Divisão Nacional de Saúde Bucal. Levantamento epidemiológico em saúde bucal: Brasil, zona urbana, 1986. Brasília (DF); 1988.

31. BRASIL - Ministério da Saúde (BR). Secretaria de Atenção à Saúde. Departamento de Atenção Básica. Projeto SB Brasil 2003: condições de saúde bucal da população brasileira 2002-2003. Resultados principais. Brasília (DF); 2004.

32. Turrell G, Sanders AE, Slade GD, Spencer AJ, Marcenes W. The independente contribution of neighborhood disadvantage and individual-level socioeconomic position to self-reported oral health: a multilevel analysis. Community Dent Oral Epidemiol. 2007; 35(3): 195-206. 
33. Damyanov ND, Witter DJ, Bronkhorst EM, Creugers NH. Dental status and associated factors in a dentate adult population in bulgaria: a cross-sectional survey. Int J Dent. 2012; 2012: 578401.

34. World Health Organization (WHO). Collaborating Centre for Oral Health Services Research University College Cork. Oral Health of Irish Adults 2000 - 2002. Cork: WHO; 2007.

35. Sanders AE, Turrell G, Slade GD. Affluent neighbourhoods reduce excess risk of tooth loss among the poor. J Dent Res. 2008; 87(10): 969-73.

36. Roncalli AG, Tsakos G, Sheiham A, Souza GC, Watt RG. Social determinants of dental treatment needs in Brazilian adults. BMC Public Health. 2014; 14: 1097-108.

37. Franco M, Diez-Roux AV, Glass TA, Caballero B, Brancati FL. Neighborhood characteristics and availability of healthy foods in Baltimore. Am J Prev Med. 2008; 35(6): 561-7.

38. Petersen PE, Lennon MA. Effective use of fluorides for the prevention of dental caries in the 21st century: the WHO approach. Community Dent Oral Epidemiol. 2004; 32(5): 319-21.

39. Neidell M, Herzog K, Glied S. The Association Between Community Water Fluoridation and Adult Tooth Loss. American Journal of Public Health. 2010; 100(10): 1980-85.

40. Guiotoku SK, Moysés ST, Moysés SJ, França BHS, Bisinelli JC. Iniquidades raciais em saúde bucal no Brasil. Rev Panam Salud Publica. 2012; 31(2): 135-41.

41. Parra FC, Amado RC, Lambertucci JR, Rocha J, Antunes CM, Pena SDJ. Color and genomic ancestry in Brazilians. Proc Natl Acad Sci USA. 2003; 100(1): 177-82.

42. Pimenta JR, Zuccherato LW, Debes AA, Maselli L, Soares RP, Moura-Neto RS, et al. Color and Genomic Ancestry in Brazilians: A Study with Forensic Microsatellites. Hum Hered. 2006; 62(4): 1905 .

43. Reisine ST. Dental disease and work loss. J Dent Res. 1984; 63(9): 1158-61.

44. Schwarz E, Hansen ER. Utilization of dental services in the adult Danish population 1975. Community Dent Oral Epidemiol. 1976; 4(6): 221-6.

45. Thomson WM, Williams SM, Broadbent JM, Poulton R, Locker D. Long-term dental visiting patterns and adult oral health. J Dent Res. 2010; 89(3): 307-11.

\section{Tables}

Table 1. Distribution of adults $(\mathrm{n}=9,564)$ according to the mean number of tooth loss. SBBrasil Project, 2010. 


\section{VARIABLES}

INTERMEDIARY DETERMINANTS

Socioeconomic position

Declared skin color

White

Education levels (in years) ${ }^{\S *}$

4049

5515

47.6

1.0

$52.4 \quad 3.0$

$(\mathrm{SD})=8.64(3.93)$

Percentile

p value

$\mathbf{n}$

$\%$ (median)
$50 \quad 75$

Family income (in US dollars)*

$\begin{array}{ccccccc}>2557 & 505 & 3.5 & 0.0 & 1.0 & 4.0 & <0.001 \dagger \\ 853-2556 & 2741 & 29.3 & 1.0 & 4.0 & 9.0 & \\ 285-852 & 4687 & 53.4 & 3.0 & 7.0 & 13.0 & \\ \leq 284 & 1404 & 13.8 & 4.0 & 8.0 & 16.0 & \end{array}$

Behavioral \& biological factors

Sex

\begin{tabular}{ccccccc}
\hline Female & 6287 & 62.3 & 2.0 & 6.0 & 12.0 & $<0.001^{* *}$ \\
\hline Male & 3277 & 37.7 & 2.0 & 5.0 & 11.0 & \\
\hline Age (in years) $^{\S}$ & $(\mathrm{SD})=39.39(3.08)$ & & & & $<0.001^{\#}$
\end{tabular}

Perception of the need for treatment*

\begin{tabular}{clllllll}
\hline Yes & 7360 & 77.0 & 2.0 & 6.0 & 12.0 & $<0.001^{* *}$ \\
\hline No & 1999 & 23.0 & 1.0 & 4.0 & 12.0 & \\
\hline Tooth pain* & & & & & & \\
\hline No & 7151 & 72.8 & 2.0 & 5.0 & 12.0 & $<0.001^{* *}$ \\
Yes & 2344 & 27.2 & 3.0 & 6.0 & 12.0 &
\end{tabular}

Health services

Previous use of dental service*

$\begin{array}{lcccccc}\text { Yes } & 8837 & 92.2 & 2.0 & 6.0 & 11.0 & 0.013^{* *} \\ \text { No } & 672 & 7.8 & 2.0 & 6.0 & 16.0 & \end{array}$

Time since the last consultation*

\begin{tabular}{lcccccc}
$\leq 1$ year & 4446 & 45.4 & 2.0 & 5.0 & 10.0 & $<0.001 \dagger$ \\
$>1$ year & 4293 & 46.8 & 2.0 & 6.0 & 13.0 & \\
\hline No previous use of dental service & 672 & 7.8 & 2.0 & 6.0 & 16.0 &
\end{tabular}

Reason for consultation*

\begin{tabular}{lcccccc} 
Review/prevention & 1910 & 19.4 & 0.0 & 3.0 & 7.0 & $<0.001 \dagger$ \\
\hline Oral health problems & 6893 & 72.8 & 3.0 & 7.0 & 12.0 & \\
\hline No previous use of dental service & 672 & 7.8 & 2.0 & 6.0 & 16.0 &
\end{tabular}

Type of dental service*

Public

Not public

No previous use of dental service

Satisfaction with dental services*

Satisfied

Dissatisfied

\begin{tabular}{cccccc}
5288 & 57.2 & 1.0 & 5.0 & 10.0 & $<0.001 \dagger$ \\
\hline 3524 & 35.0 & 3.0 & 7.0 & 13.0 & \\
\hline 672 & 7.8 & 2.0 & 6.0 & 16.0 & \\
\hline 7373 & 78.4 & 2.0 & 6.0 & 11.0 & $<0.001 \dagger$ \\
\hline 1404 & 13.8 & 3.0 & 6.0 & 12.0 &
\end{tabular}

Page 14/19 
§ Numerical variables.

* Variation at $\mathrm{n}=9,564$. Due to loss of information.

** $\mathrm{P}$ value calculated by the nonparametric Mann-Whitney test.

\# $\mathrm{P}$ value calculated by the Spearman correlation ( ()).

† P value calculated by the nonparametric Kruskall-Wallis test.

Table 2. Multilevel hierarchical negative binomial regression of the tooth loss in adults $(\mathrm{n}=$ 9,139). SBBrasil Project, 2010. 


\begin{tabular}{lcccccc}
\hline VARIABLES & Model 1 & Model 2 & \multicolumn{2}{c}{ Model 3 } \\
& MR (95\% & $\mathrm{p}$ & MR (95\% & $\mathrm{p}$ & MR (95\% & $\mathrm{p}$ \\
& $\mathrm{CI})$ & value & $\mathrm{CI})$ & value & CI) & value \\
\hline
\end{tabular}

STRUCTURAL DETERMINANTS

Socioeconomic \& political context

MHDI

Very high

High

Medium/low
Ref.

1.46 (1.19-

1.79)

$1.80(1.44-$

2.24)

Ref.

0.038

Ref.

0.001

Ref.

$<$

Yes

No

$1.15(1.01-$

1.32)

\section{INTERMEDIARY DETERMINANTS}

Socioeconomic position

Declared skin color

White

Yellow/Black/Brown/Indigenous

Education levels (in years)

Family income (in US dollars)

$$
\begin{aligned}
& >2557 \\
& 853-2556
\end{aligned}
$$$$
\text { 285-852 }
$$$$
\leq 284
$$

\section{Behavioral \& biological factors}

Sex

$$
\text { Female }
$$

Male

Age (in years)

Health services

Previous use of dental service

Yes

No

$1.24(1.09-$

1.41)

$1.27(1.11$

1.45)

Ref.

$34(1.09-\quad 0.006$

$1.46(1.17-\quad 0.001$

1.83)

0.001

1.32) 1.41)

Ref.

Ref.

$1.07(1.02-\quad 0.004$

1.12)

0.92 (0.92-

0.93)

Ref.

1.58 (1.42-

1.75)

1.89 (1.71-

2.10)

1.93 (1.72-

2.16)
1.06 (1.02-

1.11)

0.93 (0.93-

$0.94)$

0.001

Ref.

1.59 (1.44-

1.76)

1.90 (1.72-

2.10)

1.97 (1.76-

2.20)
0.007

$<$

0.001 
Reason for consultation

Review/prevention

Oral health problems

No previous use of dental

service
Ref.

$1.42(1.35-$

1.50)

$<$

$1.42(1.30-$

1.56)

$\mathrm{MR}=$ Mean ratio

Ref. $=$ Reference category

Empty model: Deviance $=59956.396$

Model 1: Deviance $=59913.184$

Model 2: Deviance $=56976.56$

Model 3: Deviance $=55016.952$

\section{Figures}

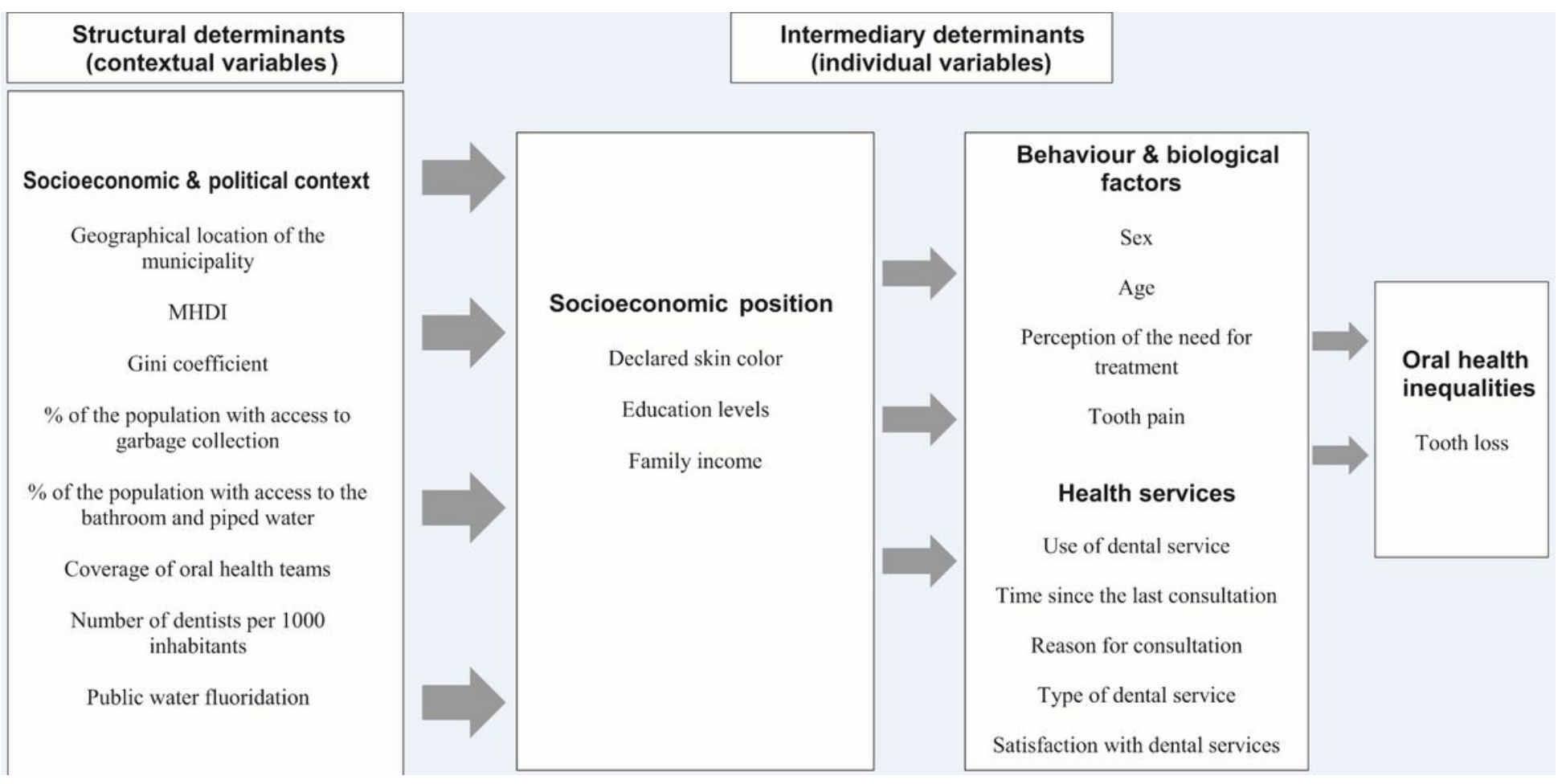

\section{Figure 1}

Conceptual model for the inequities of oral health adapted from Watt \& Sheiham (2012). 


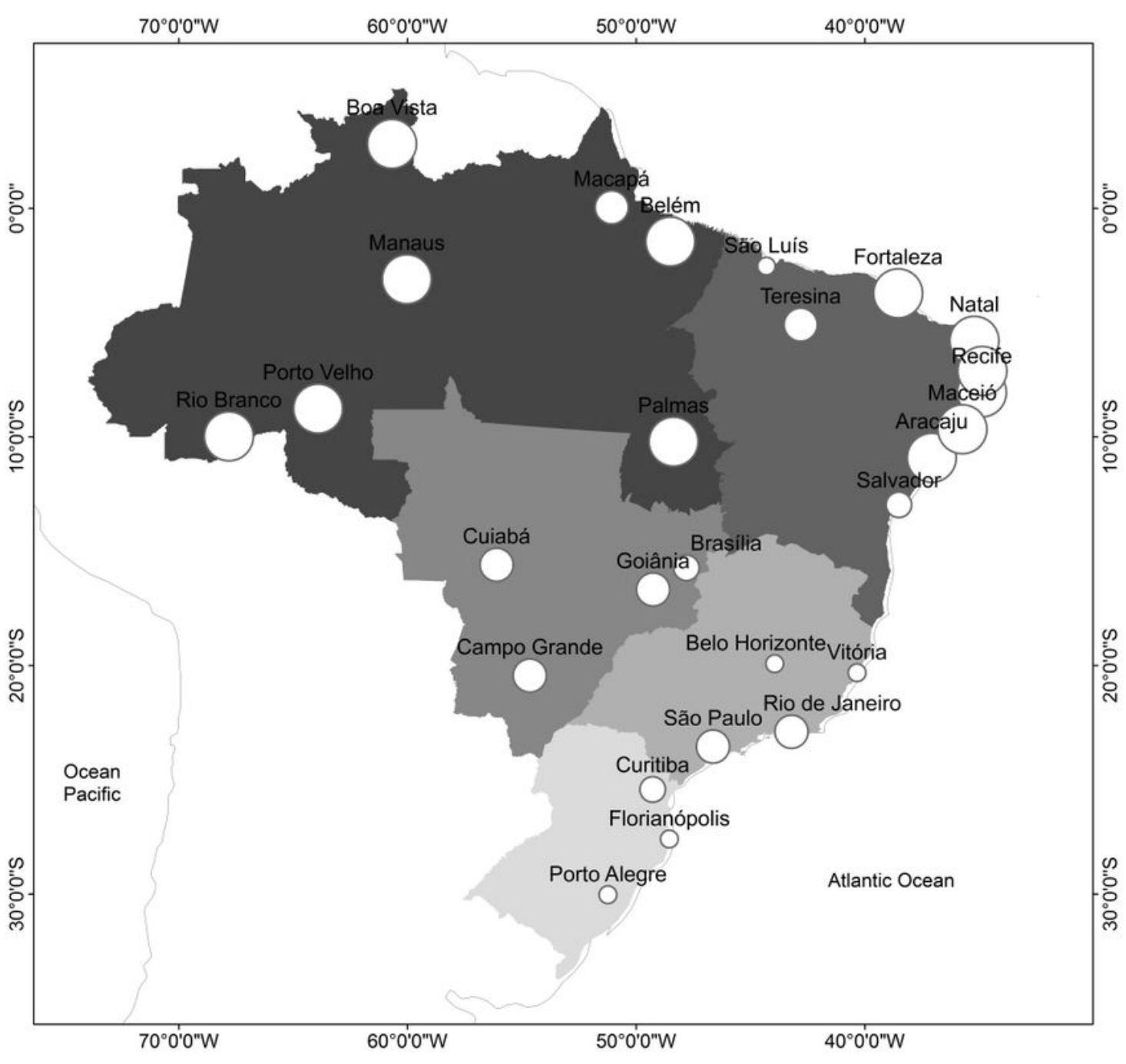

Mean $(95 \% \mathrm{Cl}) /$ region

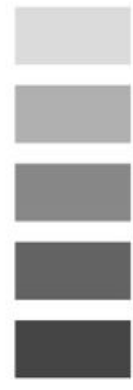

7.84 [6.75-8.93]

Mean / capital

$6.57[5.86-7.28]$

Below 6

$6,0-6,99$

$7.84[6.95-8.73]$

$7,00-7,99$

8.77 [7.81-9.73]

$10.95[10.23-11.66]$

$8,00-9,00$

Above 9

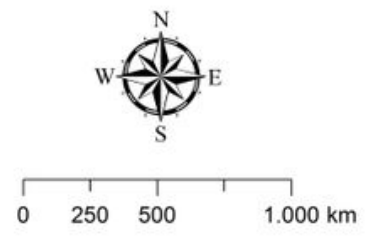

Geodetic Coordinate System - DATUM SIRGAS 2000

IBGE cartographic base, 2010.

Source: SBBrasil, 2010.

\section{Figure 2}

The mean number of missing teeth in the residents of Brazilian geopolitical macroregions $(n=9,564)$.

SBBrasil Project, 2010.

\section{Supplementary Files}


This is a list of supplementary files associated with this preprint. Click to download.

- Supplementarymaterial.docx 